\title{
Jetting during oblique impacts of spherical impactors
}

\author{
Shigeru Wakita ${ }^{\mathrm{a}, *}$, Brandon Johnson $^{\mathrm{a}}$, C. Adeene Denton ${ }^{\mathrm{a}}$, Thomas M. \\ Davison $^{\mathrm{b}}$ \\ ${ }^{a}$ Department of Earth, Atmospheric, and Planetary Sciences, Purdue University, West \\ Lafayette, IN, 47907, USA \\ ${ }^{b}$ Department of Earth Science and Engineering, Imperial College London, London, UK
}

\begin{abstract}
During the early stages of an impact a small amount material may be jetted and ejected at speeds exceeding the impact velocity. Jetting is an important process for producing melt during relatively low velocity impacts. How impact angle affects the jetting process has yet to be fully understood. Here, we simulate jetting during oblique impacts using the iSALE shock physics code. Assuming both the target and impactor have the same composition (dunite), we examine the jetted material which exceeds the impact velocity. Our results show that oblique impacts always produce more jetted ejecta than vertical impacts, except for grazing impacts with impact angles $<15^{\circ}$. A $45^{\circ}$ impact with an impact velocity of $3 \mathrm{~km} / \mathrm{s}$ produces jetted material equal to $\sim 7 \%$ of the impactor mass. This is 6 times the jetted mass produced by a vertical impact with similar impact conditions. We also find that the origin of jetted ejecta depends on impact angle; for impact angles less than $45^{\circ}$, most of the jet is composed of impactor material, while at higher impact angles the jet is dominated by target material. Our findings are consistent with previous experimental work. In all cases, jetted materials are preferentially distributed downrange of the impactor.
\end{abstract} Keywords: Impact processes, Collisional physics, Asteroids

*Corresponding author 


\section{Introduction}

Impact jetting occurs during the earliest stage of impact cratering when the impactor is still making contact with the target (e.g., Birkhoff et al., 1948, Walsh et al. 1953). The jet contains the first material ejected, is highly shocked, and is ejected at speed exceeding the impact velocity. The total mass of jetted ejecta is small, typically less than a few percent of the impactor mass. Impact jetting has been proposed as a mechanism for producing tektites (Vickery, 1993, Stöffler et al. 2013), producing melt in lightly shocked material during grain-on-grain collision (Kieffer, 1977), and producing chondrules (Kieffer, 1975, Johnson et al. 2015 Wakita et al., 2017). Jetting has also been proposed as a critical component of vaporization during massive impacts (Melosh and Sonett, 1986), and to affect the final ice-rock-ratio of icy bodies (McKinnon, 1989a|b). However, jetting, especially during oblique impacts, remains poorly understood.

Experimental and theoretical studies have explored jetting during planetary impacts using thin plate theory (e.g., Kieffer, 1975; Melosh and Sonett, 1986 Vickery, 1993). Thin plate theory describes jetting during the symmetric collision of two thin plates and can predict the jet velocity and the critical angle above which jetting occurs as a function of plate material and impact velocity (Birkhoff et al., 1948: Walsh et al., 1953). However, thin plate theory fails when applied to planetary impacts, which are better described by the impact between a spherical impactor and a plate than of two thin plates, including predictions of ejecta temperature and the ratio of target to impactor material in jets produced by spherical impactors (Sugita and Schultz, 1999). Numerical models (Johnson et al. 2014) and laboratory experiments (Kurosawa et al., 2015) show that the maximum velocities of jets produced during the impact of spherical bodies are about half the velocity predicted by thin plate theory.

Vickery (1993) estimated that very oblique impacts could have up to ten times more jetted material than vertical impacts with the same impact velocity. While these estimates are based on thin plate theory, which does not accurately describe the jetting physics during impact of spherical bodies (Yang and 
Ahrens, 1995, Kurosawa et al., 2015), oblique impacts should produce more jetted material than head-on collisions as experimental results of the spherical impactor indicate (Sugita and Schultz, 1999). However, without direct simulation in three dimensions, we cannot quantify how impact angle will affect the amount of jetted material produced by a given impact.

Most impacts are believed to be oblique; a typical impact angle is expected to be $45^{\circ}$ (Shoemaker, 1962). The effect of impact angle on crater formation has been studied experimentally (e.g., Gault and Wedekind, 1978, Schultz and Gault, 1990, Burchell and Mackay, 1998) and numerically (e.g., Davison et al., 2011, Elbeshausen et al., 2009). Highly oblique impacts can produce elongated craters as found on Mars, Venus, and the Moon (Bottke et al. 2000). Even moderately oblique impacts have a significant effect on distal impact ejecta, producing a wedge of avoidance in the uprange direction (Gault and Wedekind, 1978), and elongated craters produced by grazing impacts exhibit in a butterflylike distal ejecta pattern (Gault and Wedekind, 1978; Schultz and Gault, 1990. Speyerer et al. 2016). How impact angle affects the distribution of the very fastest and earliest ejecta remains uncertain. Here, we simulate oblique impacts to determine how impact angle affects the jetting process. This includes determining how the amount and distribution of jetted material depends on impact angle. In Section 2, we describe the setup for our numerical simulations. We then present results from our simulations (Section 3) and discuss the implications for our understanding of the unique physics surrounding jetted material during impact (Section 4).

\section{Methods}

We simulate jetting during oblique impacts using the iSALE-3D shock physics code, which includes both a strength model and the capability to simulate porous compaction (Hirt et al., 1974, Collins et al., 2004, Melosh et al., 1992; Ivanov et al., 1997; Wünnemann et al. 2006; Collins et al., 2011). We assume that our spherical impactor and flat target have the same dunite composition $\overline{B e n z}$ 
et al. 1989), as used in previous jetting work (e.g., Johnson et al., 2014). We note these results are only strictly valid for a dunite impactor and target. More compressible materials are known to have a lower jetting efficiency (Johnson et al. 2014). Jetting during the impact of a projectile with distinct composition from the target will also affect jetting dynamics (Sugita and Schultz, 1999). We expect, however, that the downrange momentum of the impactor in oblique impacts will still tend to enhance jetting regardless of composition. The material properties for dunite used in iSALE-3D are described in Table 1.

In our simulations, we vary impact angle $\left(\theta_{\text {imp }}\right)$ and impact velocity $\left(v_{\text {imp }}\right)$. Impact angle in our simulations is measured from the horizontal surface of the target, such that $\theta_{\mathrm{imp}}=90^{\circ}$ is a vertical impact. We simulate a range of impact angles from $15^{\circ}$ to $90^{\circ}$ in $15^{\circ}$ increments, with impactor velocities of $2 \mathrm{~km} / \mathrm{s}, 3$ $\mathrm{km} / \mathrm{s}$, and $5 \mathrm{~km} / \mathrm{s}$. This range of velocities spans the expected range of impact velocities during planetesimal accretion, which depends on the size of the target bodies (i.e., the escape velocity of the system, Kokubo and Ida, 2000, Johnson et al., 2015; Hasegawa et al. 2016). This is also the velocity range where the jetted mass is highest for vertical impacts of dunite impactors on dunite targets (Johnson et al. 2015, Wakita et al., 2017).

We record the behavior of jetted ejecta in iSALE-3D using Lagrangian tracer particles to track material position, velocity, pressure, and temperature. Note that the tracer particles, which track the motion of a parcel of material, are initially placed at the center of each cell and we calculate their mass using their initial spacing, locations, and density (e.g., Johnson and Melosh, 2014). We focus on tracer particles since they allow us to determine material provenance as well as mass. High resolution is required to accurately evaluate the velocities, ejection angles, and mass of jetted ejecta. We use a fixed impactor diameter of $D_{\mathrm{imp}}=1 \mathrm{~km}$, with a spatial resolution of $5 \mathrm{~m}$ or 100 cells per projectile radius. Previous work showed that for 10-km-diameter bodies, jetting occurs in a near-surface zone at most $\sim 400 \mathrm{~m}$ deep (Wakita et al., 2017). As such, the high-resolution zone of our model incorporates the lower half of the impactor and a thin surface layer of target material. We use this setting for most of our 
runs. iSALE-3D divides simulations into a high-resolution zone and an extension zone; in the extension zone cell size increases with distance from the highresolution zone. To avoid errors introduced by motion through the extension zone, we only track tracer particles in the high resolution zone. To confirm that our high-resolution zone appropriately resolves the jetting process, we compare our iSALE-3D results of vertical impacts with iSALE-2D runs using the same resolution Appendix A . We also ensure that all jetted material originates from and is tracked within the high resolution zone. We confirm that a high resolution zone encompassing half of the impactor resolves jetted material well for most oblique impact scenarios. For some oblique impact scenarios $\left(\theta_{\text {imp }}=30^{\circ}\right.$ at $v_{\text {imp }}=5 \mathrm{~km} / \mathrm{s}$ and $\theta_{\text {imp }}=45^{\circ}$ at $v_{\text {imp }}=2,3$, and $5 \mathrm{~km} / \mathrm{s}$ ), we enlarge the highresolution zone in the vertical direction to include all jetted impactor material. 
Table 1: iSALE input parameters

\begin{tabular}{|c|c|}
\hline Description & Values \\
\hline Equation of state & ANEOS \\
\hline Bulk material of impactor/target & dunite $^{a}$ \\
\hline Solidus temperature & $1373 \mathrm{~K}^{\mathrm{b}}$ \\
\hline Simon approximation constant A & $1520 \mathrm{MPa}^{\mathrm{c}}$ \\
\hline Simon approximation exponent C & $4.05^{\mathrm{c}}$ \\
\hline Poisson's ratio & $0.25^{\mathrm{d}}$ \\
\hline Thermal softening parameter & $1.1^{\mathrm{d}}$ \\
\hline Strength model & Rock $^{\text {e }}$ \\
\hline Cohesion (damaged) & $0.01 \mathrm{MPa}^{\mathrm{d}}$ \\
\hline Cohesion (undamaged) & $10 \mathrm{MPa}^{\mathrm{d}}$ \\
\hline Frictional coefficient (damaged) & $0.6^{\mathrm{d}}$ \\
\hline Frictional coefficient (undamaged) & $1.2^{\mathrm{d}}$ \\
\hline Strength at infinite pressure & $3.5 \mathrm{GPa}^{\mathrm{d}}$ \\
\hline Damage model & Ivanov e \\
\hline Minimum failure strain & $10^{-4 \mathrm{~d}}$ \\
\hline Damage model constant & $10^{-11 \mathrm{~d}}$ \\
\hline Threshold pressure for damage model & $300 \mathrm{MPa}^{\mathrm{d}}$ \\
\hline \multicolumn{2}{|l|}{ a Benz et al. (1989) } \\
\hline \multicolumn{2}{|l|}{ b Davison et al. (2016) } \\
\hline \multicolumn{2}{|l|}{ c Davison (2010) } \\
\hline d Kurosawa and Genda 2018) & \\
\hline e Collins et al. (2004) & \\
\hline
\end{tabular}

\section{Results}

\subsection{Effect of impact angle on distribution of jetted material}

Figure 1 shows a time series of our fiducial model, an oblique impact with $\theta_{\mathrm{imp}}=45^{\circ}$ and $v_{\mathrm{imp}}=3 \mathrm{~km} / \mathrm{s}$. Each panel represents $t=0.0 t_{s}, 0.25 t_{s}$, 

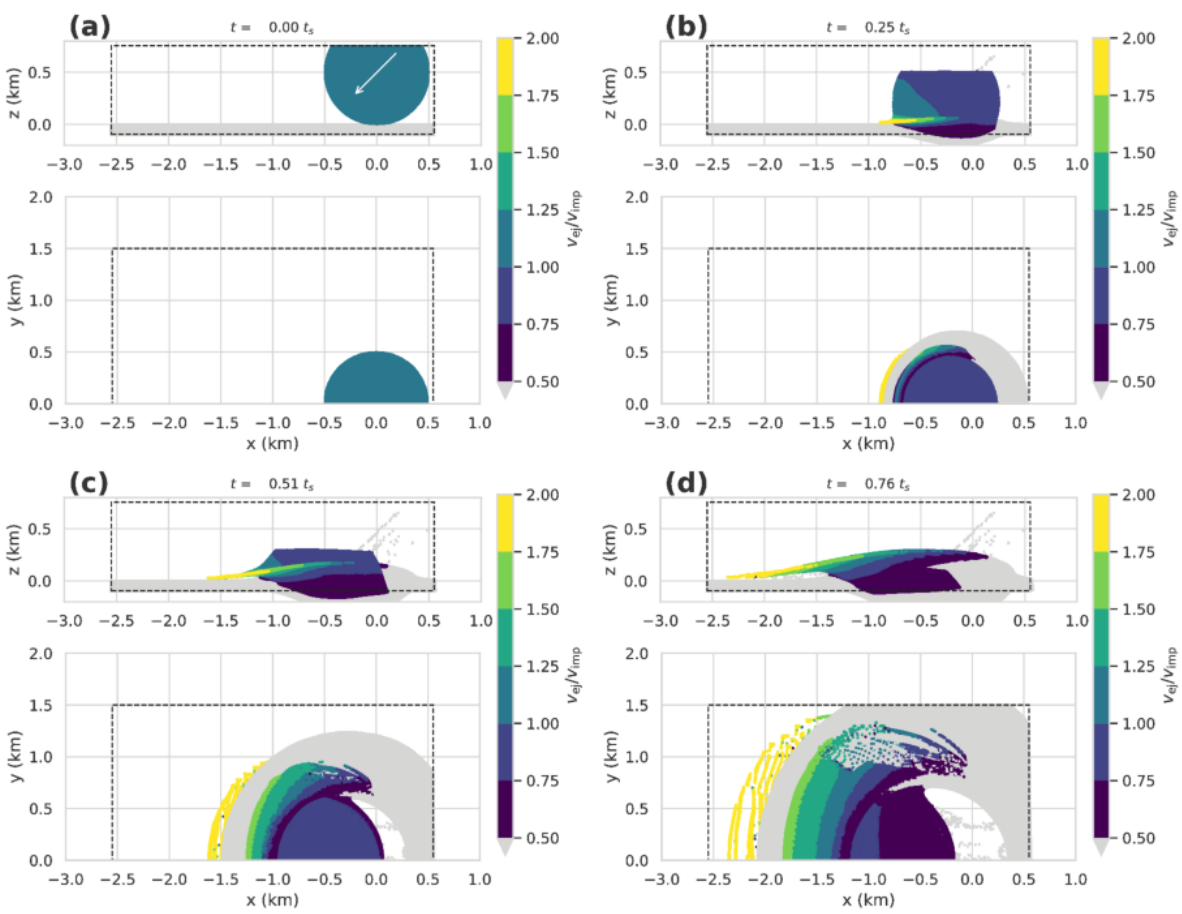

Figure 1: Time series for our fiducial model $\left(v_{\mathrm{imp}}=3 \mathrm{~km} / \mathrm{s}\right.$ and $\left.\theta_{\mathrm{imp}}=45^{\circ}\right)$. Color represents tracer particle velocity $\left(v_{\mathrm{ej}}\right)$ normalized by the impact velocity. The top panels are a cross-sectional view, while the bottom panels are a top view on the impact from above. Each panel represents (a) $t=0.0 t_{s}$, (b) $t=0.25 t_{s}$, (c) $t=0.51 t_{s}$, and (d) $0.76 t_{s}$ after the impact, respectively. White arrow in top-left panel shows the direction of impactor. Note the impactor is spherical, but only contains tracer particles in lower part, due to our setting of high resolution zone (shown as dashed line). 
$0.51 t_{s}$, and $0.76 t_{s}$ after the impact, respectively, where $t_{s}$ is the characteristic time for contact and compression defined by $t_{s}=D_{\mathrm{imp}} /\left(v_{\mathrm{imp}} \sin \left(\theta_{\mathrm{imp}}\right)\right)$. The tracer particles shown in Figure 1 reflect our setup of high-resolution zone, which includes the lower portion of the spherical impactor and the near-surface portion of the target. Figure 2 illustrates only the tracer particles whose velocity is higher than the half of the impact velocity with a positive velocity in vertical direction. Faster ejecta $\left(v_{\mathrm{ej}} / v_{\mathrm{imp}}>1\right)$ is focused in the downrange direction and ejected at very low angles as evidenced by its nearly horizontal motion. The higher velocity ejecta $\left(v_{\mathrm{ej}} / v_{\mathrm{imp}}>1.6\right)$ are preferentially distributed downrange and originate from the impactor.

Figure 3 illustrates the relationship between ejecta velocity and azimuthal ejection angle as defined with respect to the orientation of the impactor (downrange is $0^{\circ}$, uprange $\left.180^{\circ}\right)$. As Figure 3 indicates, jetted ejecta $\left(v_{\mathrm{ej}} / v_{\mathrm{imp}}>1\right)$ is largely distributed downrange, with azimuths less than $90^{\circ}$. Azimuthal angle shallows as the ejecta velocity increases. Because the impactor is traveling in the downrange direction on impact, downrange ejecta acquires higher velocity than ejecta not aligned with the impactor. Although the distribution of ejecta produced from the target and the impactor in Figure 2 is similar, the maximum velocity of ejecta produced from impactor material is $v_{\mathrm{ej}} / v_{\mathrm{imp}}=2$, which is higher than ejecta produced from the target of $v_{\mathrm{ej}} / v_{\mathrm{imp}}=1.6$.

Figures 4,5 and 6 show the resulting high-velocity ejecta trends for impact scenarios incorporating $\theta_{\mathrm{imp}}=90^{\circ}, 60^{\circ}, 30^{\circ}$, and $15^{\circ}$. For a head-on impact $\left(\theta_{\mathrm{imp}}=90^{\circ}\right)$ ejecta are distributed symmetrically from the impact point (defined as the origin, 0, 0) (Figures 4a , 5a, 6a). Ejecta behavior for $\theta_{\mathrm{imp}}=60^{\circ}$ is similar to $\theta_{\text {imp }}=45^{\circ}$, our fiducial case (Figures $4 \mathrm{~b}, 5 \mathrm{~b}$ and $6 \mathrm{p}$ ); however, as impact angle continues to decrease $\left(\theta_{\mathrm{imp}}=30^{\circ}\right)$, the high-velocity portion of ejecta $\left(v_{\mathrm{ej}} / v_{\mathrm{imp}}>1.2\right)$ becomes bimodally distributed and material is predominately jetted either immediately downrange $\left(0^{\circ}\right)$ or at an angle of $\sim 40^{\circ}$ (Figures 5 ; and 6. ). Note that ejection angles in iSALE-3D have some preferences, such as $45^{\circ}$ and $135^{\circ}$ (Figure 6a), due to the use of cartesian coordinates. While such a bimodal distribution is only observed for a $30^{\circ}$ impact, this behavior may be 

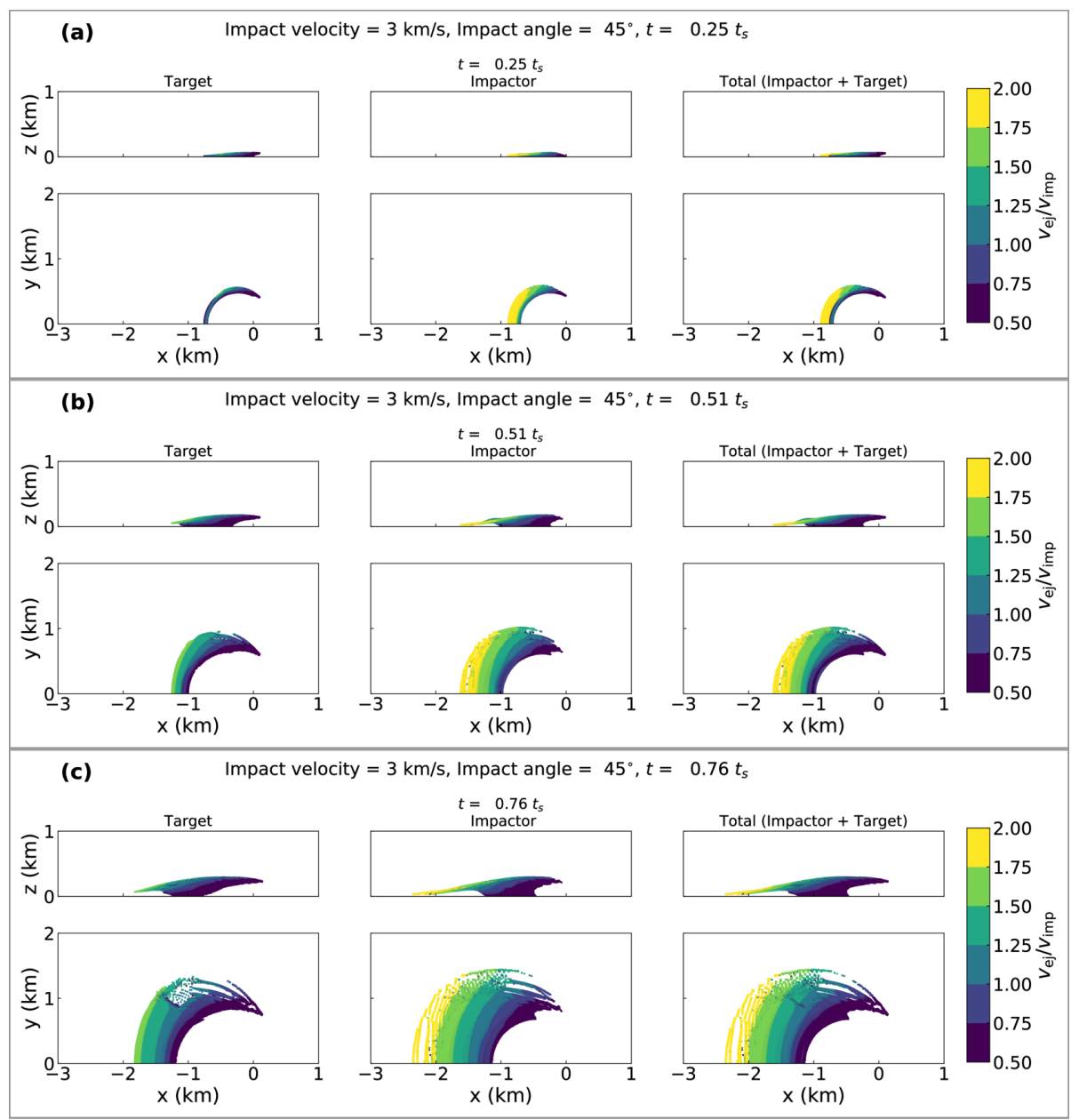

Figure 2: Material ejected at (a) $t=0.25 t_{s}$, (b) $t=0.51 t_{s}$, and (c) $t=0.76 t_{s}$ for our fiducial model $\left(v_{\mathrm{imp}}=3 \mathrm{~km} / \mathrm{s}\right.$ and $\left.\theta_{\mathrm{imp}}=45^{\circ}\right)$. Color represents ejecta velocity $\left(v_{\mathrm{ej}}\right)$ normalized by the impact velocity. Top panels are a cross-sectional view, while the bottom panels are a bird's eye view on the impact from above. Ejecta produced by the target are shown on the left, ejecta from the impactor in the middle, and the total ejecta produced from the impact on the right, respectively. 

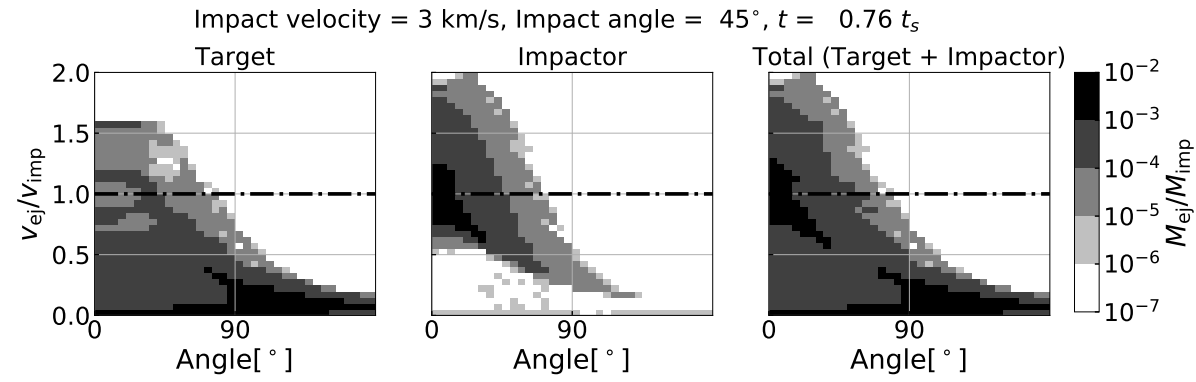

Figure 3: Heatmap of ejecta density as a function of ejection angle and velocity for our fiducial model $\left(v_{\mathrm{imp}}=3 \mathrm{~km} / \mathrm{s}\right.$ and $\left.\theta_{\mathrm{imp}}=45^{\circ}\right)$. Ejection angle is defined with respect to the orientation of the oblique impactor (downrange is $0^{\circ}$, uprange $180^{\circ}$ ). Note ejection angle is measured in the azimuthal direction, not in vertical direction (elevation angle). Gray contours represent mass of ejecta $M_{\mathrm{ej}}$ normalized by mass of impactor $M_{\mathrm{imp}}$ : each bin of ejection angle and velocity represents the amount of ejecta in the bin (the dark-colored bin has more ejecta than the light-colored bin). Note that angle bins are 5 degree wide and velocity bins have a width of $0.05 v_{\mathrm{ej}} / v_{\mathrm{imp}}$. Ejecta produced by the target are shown on the left, ejecta from the impactor in the middle, and the total ejecta produced from the impact on the right, respectively.

similar to the distal distribution of ejecta around elongated craters on Moon and Mars, which originate from oblique impacts (Gault and Wedekind, 1978 . Schultz and Lutz-Garihan, 1982). The volume of ejecta produced for a grazing impact $\left(\theta_{\mathrm{imp}}=15^{\circ}\right)$ scenario appears minimal (Figures $5 \mathrm{~d}$ and 6 $\mathrm{d}$ ), and highvelocity ejecta $\left(v_{\mathrm{ej}} / v_{\mathrm{imp}}>0.5\right)$ is only distributed downrange of the impact site. We find almost all of high-velocity ejecta originate from the impactor.

\subsection{Amount of jetted ejecta in oblique impacts}

We use Lagrangian tracer particles to quantify the amount of jetted ejecta for different impact angle and impact velocity scenarios. We define tracer particles as jetted ejecta when the tracer velocity exceeds the impact velocity, the vertical component of tracer velocity is positive, and the material is located above the surface of the target. Following the evolution of tracer particles that satisfy these conditions, our analysis indicates that as the impact progresses more material is ejected until jetting ceases. The earliest ejecta is the fastest 

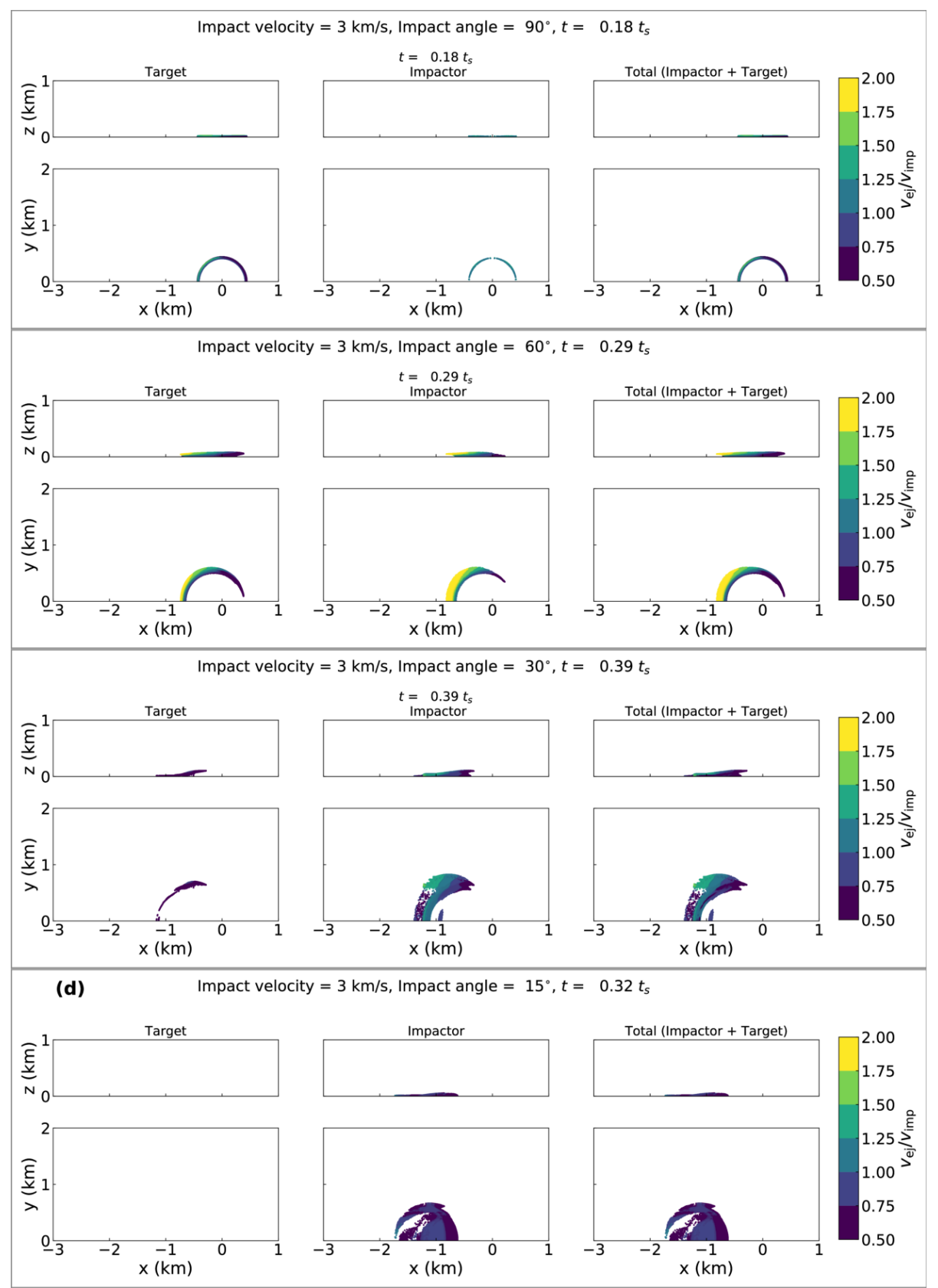

Figure 4: Behavior of ejected material for $\theta_{\mathrm{imp}}=90^{\circ}, 60^{\circ}, 30^{\circ}$, and $15^{\circ}$ (top to bottom). Same color scheme and viewing geometries as Figure 2 


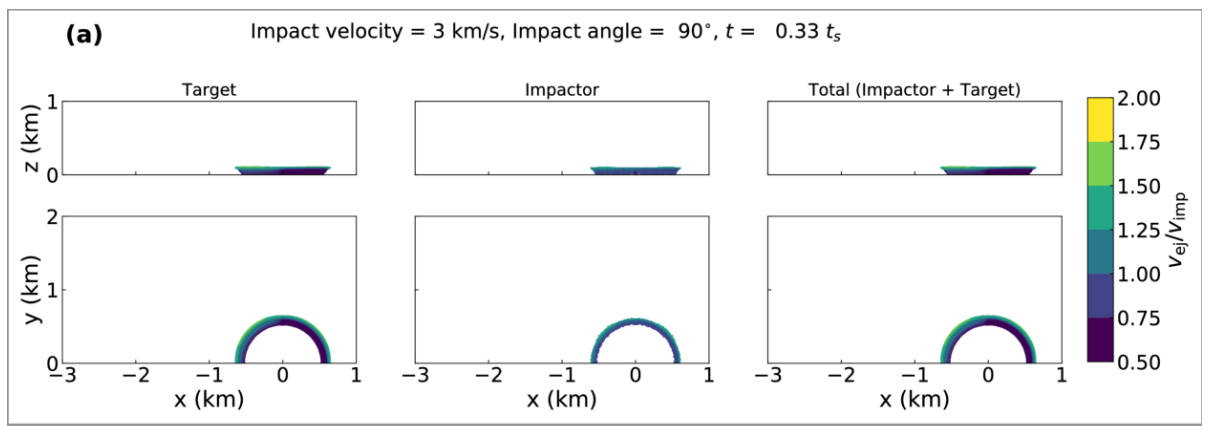

(b)
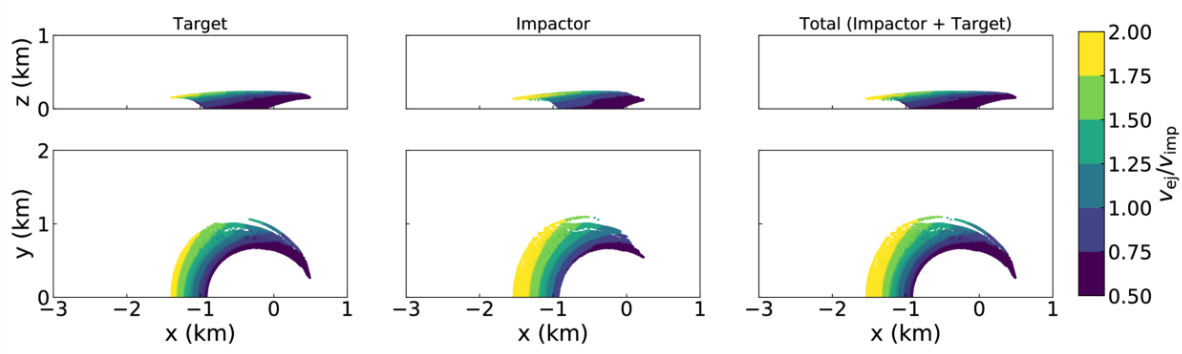

(c)
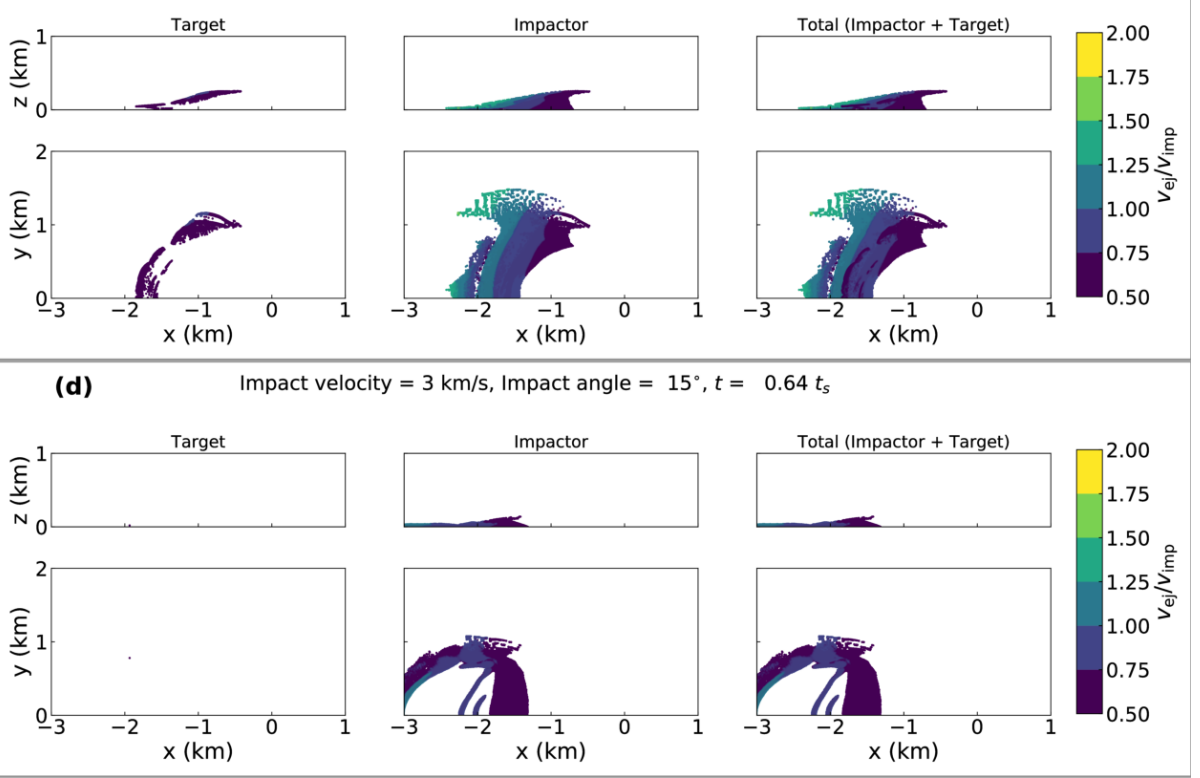

Figure 5: Same as Figure 4 but at later time. 

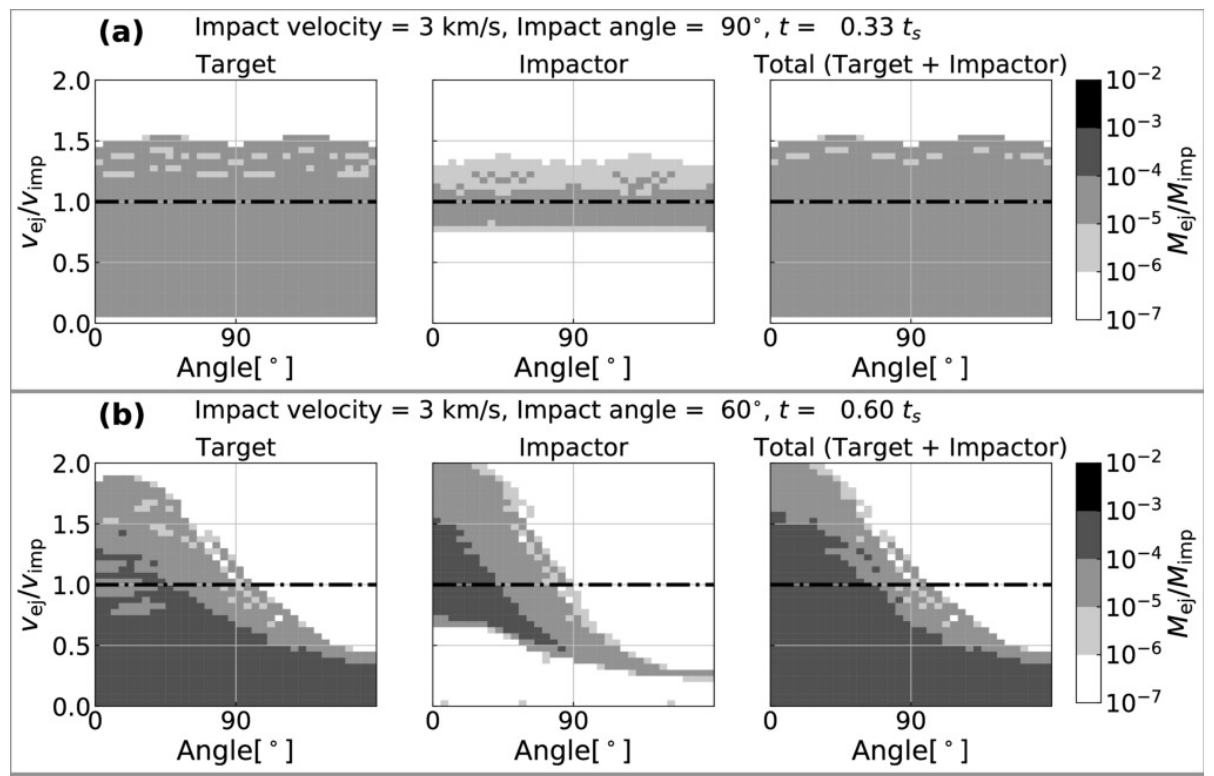

(c) Impact velocity $=3 \mathrm{~km} / \mathrm{s}$, Impact angle $=30^{\circ}, t=0.78 t_{s}$

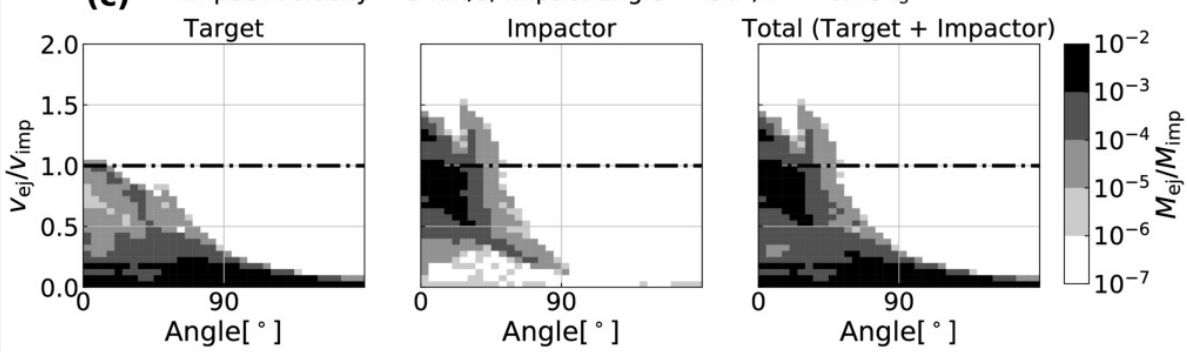

(d) Impact velocity $=3 \mathrm{~km} / \mathrm{s}$, Impact angle $=15^{\circ}, t=0.64 t_{s}$

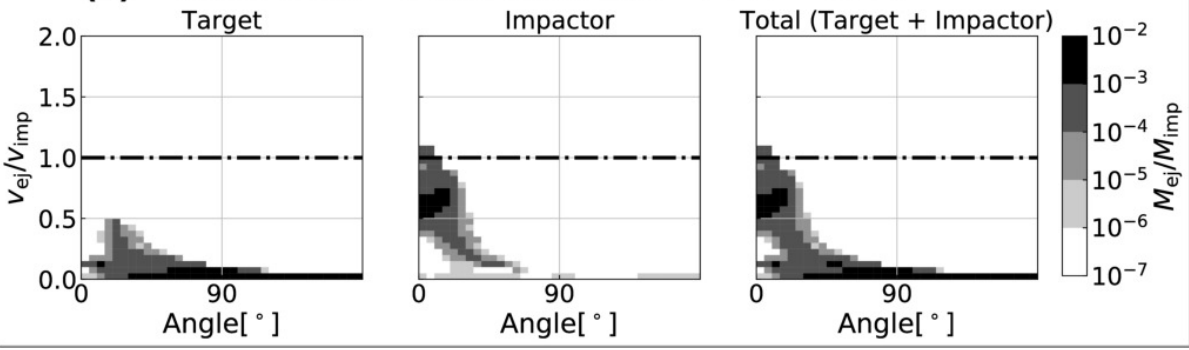

Figure 6: Same color scheme and measurements as Figure 3 produced for $\theta_{\text {imp }}=90^{\circ}, 60^{\circ}$, $30^{\circ}$, and $15^{\circ}$ (top to bottom). 


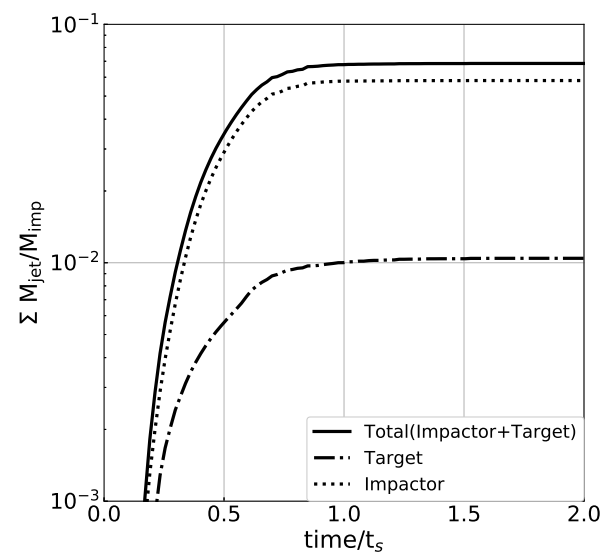

Figure 7: Evolution of cumulative jetted material mass over time for our fiducial model $\left(v_{\mathrm{imp}}=3 \mathrm{~km} / \mathrm{s}\right.$ and $\left.\theta_{\mathrm{imp}}=45^{\circ}\right)$. Each line represents jetted mass sourced from the target (dotted-dashed line), the impactor (dotted line), and the total amount of material ejected (solid line), respectively.

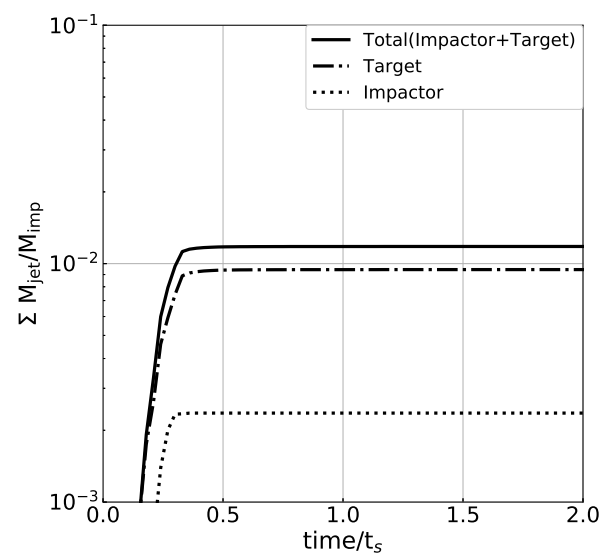

Figure 8: Additional plots of jetted material mass over time as in Figure 7 but for $\theta_{\text {imp }}=$ $90^{\circ}$. 


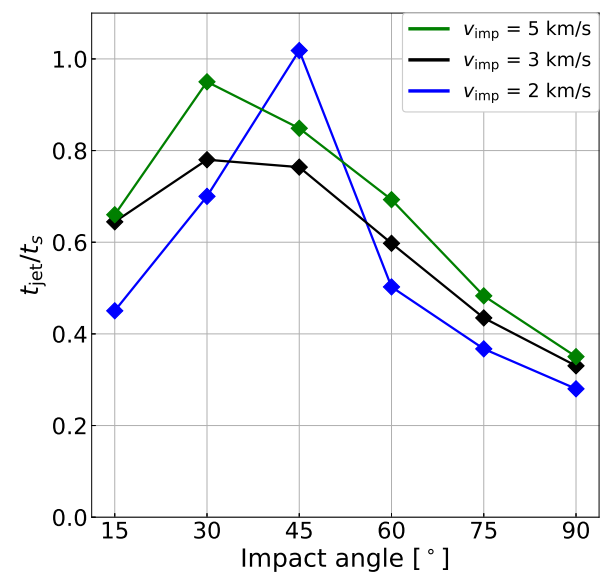

Figure 9: Time of jetting $\left(t_{\text {jet }}\right)$ as a function of impact angles. Color represent different impact velocities: $2 \mathrm{~km} / \mathrm{s}$ (blue), $3 \mathrm{~km} / \mathrm{s}$ (black), and $5 \mathrm{~km} / \mathrm{s}$ (green).

while the latest is ejected just above the impact velocity. To calculate for the cumulative jetted mass, we sum all tracer particles that fit this definition of jetted ejecta during impact. Figure 7 illustrates the evolution of the cumulative jetted mass $M_{\text {jet }}$ normalized by the impactor mass $M_{\text {imp }}$ over time for our fiducial model $\left(v_{\mathrm{imp}}=3 \mathrm{~km} / \mathrm{s}\right.$ and $\left.\theta_{\mathrm{imp}}=45^{\circ}\right)$. The jetted material reaches $90 \%$ of the total cumulative mass at $t=0.76 t_{s}$. However, for a head-on impact, this peak occurs much earlier as $t=0.33 t_{s}$ (Figure 8); this delay in jetting shows that impact jetting occurs over longer timescales for oblique impacts $\left(\right.$ note $t_{s}=D_{\text {imp }} /\left(v_{\text {imp }} \sin \left(\theta_{\text {imp }}\right)\right)$ ). Figure 9 represents the time of jetting $t_{\text {jet }}$, when $\Sigma M_{\text {jet }}$ reaches $90 \%$ of its final value, as a function of impact angle. Even when scaled by the contact and compression timescale, the jetting timescale initially increases as impact angle decreases at a given impact velocity. This is likely due to the downrange momentum of the impactor and a favorable, less asymmetric, geometry for jetting. Simulations without material strength show this trend continues to $\theta_{\mathrm{imp}}=30^{\circ}$ for all impact velocities, demonstrating that material strength causes jetting to cease earlier in the case of $\theta_{\mathrm{imp}}=30^{\circ}$. The jetting timescale for $\theta_{\mathrm{imp}}=15^{\circ}$ is shorter than other oblique impacts (Figure 97. For grazing impacts downward velocity is reduced before the contact 

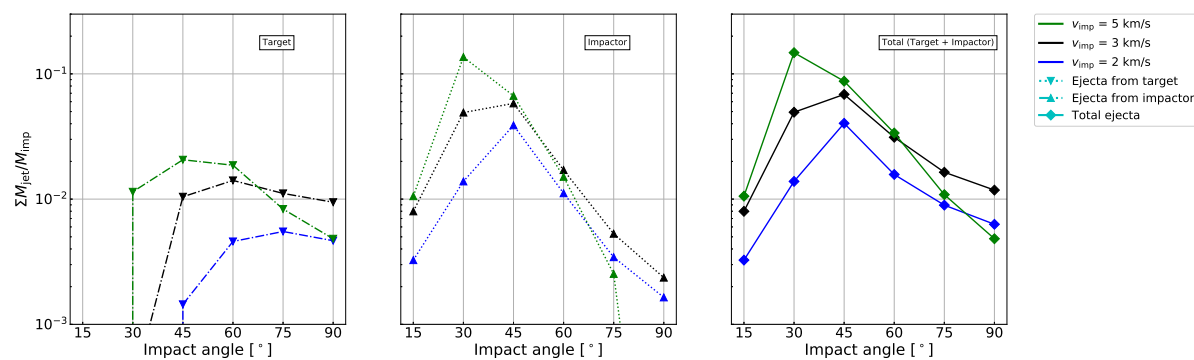

Figure 10: Mass of jetted material $\left(M_{\text {jet }}\right)$ as a function of impact angles. Color represent different impact velocities: $2 \mathrm{~km} / \mathrm{s}$ (blue), $3 \mathrm{~km} / \mathrm{s}$ (black), and $5 \mathrm{~km} / \mathrm{s}$ (green). Each panel depicts the total mass $M_{\text {jet }}$ normalized by $M_{\text {imp }}$ from the target (left), impactor (middle), and the total mass (right), respectively.

and compression phase is over, which sometimes results in decapitation of the impactor (Schultz and Gault, 1990, Davison et al., 2011). When this occurs, the impactor does not penetrate the target and jetting ceases earlier than in moderately oblique impacts.

Figure 10 summarizes the trends in cumulative jetted material mass $\Sigma M_{\text {jet }}$ for all impact scenarios. Each panel represents $\Sigma M_{\text {jet }}$ separated by material origin: material originating from the target (dotted-dashed lines, left), impactor (dotted line, middle), and the total mass (solid line, right), respectively. We first focus on the results of $v_{\mathrm{imp}}=3 \mathrm{~km} / \mathrm{s}$ (black lines), our fiducial velocity. In this scenario, almost all oblique impacts $\left(\theta_{\mathrm{imp}}=75^{\circ}, 60^{\circ}, 45^{\circ}\right.$, and $\left.30^{\circ}\right)$ produce more jetted ejecta than the head-on impacts: $1.6 \%, 3.1 \%, 6.8 \%$, and $4.9 \%$ jetted mass normalized by impactor mass $\left(M_{\mathrm{imp}}\right)$, respectively, in comparison to 1.1 $\%$ for a vertical impact. Jetted ejecta from our grazing impact $\left(\theta_{\mathrm{imp}}=15^{\circ}\right)$ in this scenario is less than $1 \%$ (Figures $5 \mathrm{~d}$ and $6 \mathrm{~d}$ ). $\theta_{\mathrm{imp}}=45^{\circ}$ produces the most jetted mass $(\sim 7 \%)$.

The cumulative jetted mass in our fiducial velocity scenario $\left(v_{\mathrm{imp}}=3 \mathrm{~km} / \mathrm{s}\right)$ depends on impact angle, which also influences the source of jetted material (Figure 10). As Figure 7 indicates, material from the impactor dominates the cumulative mass of jetted ejecta in our fiducial model $\left(v_{\mathrm{imp}}=3 \mathrm{~km} / \mathrm{s}\right.$ and $\theta_{\mathrm{imp}}$ $\left.=45^{\circ}\right)$. In contrast, jetted ejecta is dominated by target material for head-on 


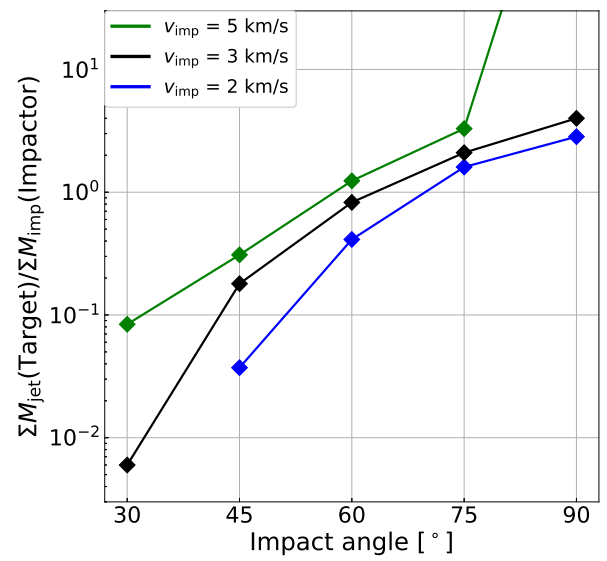

Figure 11: Mass ratio of jetted material from the target to the impactor as a function of impact angles. Note that no symbols (e.g., $15^{\circ}$ cases) means no jetted material (see Figure 10 .

impacts (Figure 8); this distinction may result from the downrange momentum of the oblique impactor. The fraction of impactor to the total jetted mass for our nominal impact velocity is $84 \%$ for a $45^{\circ}$ impact and $20 \%$ for a head-on impact. This trend is consistent with the results of laboratory experiments in previous work (Schultz and D'Hondt, 1996, Sugita and Schultz, 1999). These experiments showed that oblique impacts tend to produce more jetted material from the impactor. Our results are consistent with this trend: Figure 11 clearly illustrates that the ratio of jetted material from the target to the impactor decreases as the impact angle gets shallower.

Our results for a lower impact velocity $\left(v_{\mathrm{imp}}=2 \mathrm{~km} / \mathrm{s}\right.$, blue lines in Figure 10 indicate that $\Sigma M_{\text {jet }}$ also depends on impact velocity. In this scenario, the jetted mass produced in head-on impacts is $0.6 \%$ of the impactor mass, almost half of that produced for $v_{\mathrm{imp}}=3 \mathrm{~km} / \mathrm{s}$. Oblique impacts also produce less jetted ejecta at a lower velocity; however, we see similar trends with impact angle and $\theta_{\mathrm{imp}}=45^{\circ}$ still produces the largest mass of jetted material (4.0\% of the impactor mass). Since the jetting timescale of $45^{\circ}$ impact is much longer than other impacts with $v_{\text {imp }}=2 \mathrm{~km} / \mathrm{s}$ (Figure 9), jetting in $\theta_{\text {imp }}=45^{\circ}$ continues 
longer then produces more jetted mass. A lower jetted mass at a lower $(2 \mathrm{~km} / \mathrm{s})$ impact velocity contradicts previous simulations using a strengthless material (Johnson et al. 2014), likely because our incorporation of material strength limits the amount of jetted ejecta produced by low velocity impacts. This behavior is supported by previous modeling work (Johnson et al., 2015, Wakita et al. 2017, see also Appendix A.

When $v_{\text {imp }}$ is increased $\left(v_{\text {imp }}=5 \mathrm{~km} / \mathrm{s}\right.$, green lines in Figure 10$)$, the resulting behavior of the jetted ejecta can be explained by two competing effects. As impact velocity increases, jetting initiates later (e.g., Sugita and Schultz, 1999 Johnson et al., 2014). For $\theta_{\text {imp }}=90^{\circ}$ and $75^{\circ}$ this effect reduces the amount of jetted material as compared to our simulations with $v_{\text {imp }}=3 \mathrm{~km} / \mathrm{s}$. While the time when jetting ceases is almost the same for $\theta_{\mathrm{imp}}=90^{\circ}$ and $75^{\circ}$ regardless of impact velocity, jetting ceases earlier for lower-velocity impacts occurring at lower impact angles (Figure 91). Thus, despite increasing impact velocity, jetted mass for $\theta_{\mathrm{imp}}=45^{\circ}$ and $60^{\circ}$ are similar to the fiducial case. As impact angle is decreased $\left(\theta_{\text {imp }}=30^{\circ}\right)$, the time of jetting increases and more jetted mass is produced than our fiducial case ( $14 \%$ compared to $6.8 \%$ ), which is the highest value among impact with $v_{\mathrm{imp}}=5 \mathrm{~km} / \mathrm{s}$. Simulations without material strength show that jetted mass decreases as impact velocity increases regardless of impact angle, which is consistent with previous work (Johnson et al. 2014). Thus, material strength has an important effect on the jetting efficiency during oblique impacts in the velocity range explored.

\section{Discussion}

Our results show that the efficiency and dynamics of the jetting process are sensitive to impact angle and impact velocity. In general, oblique impacts tend to produce less shock-heated material than vertical impacts (Pierazzo and Melosh, 2000, Davison et al., 2014). However, this trend does not hold when considering only jetted material. Previous work suggests that the fraction of jetted material that reaches high temperatures (i.e., may experience melting and 

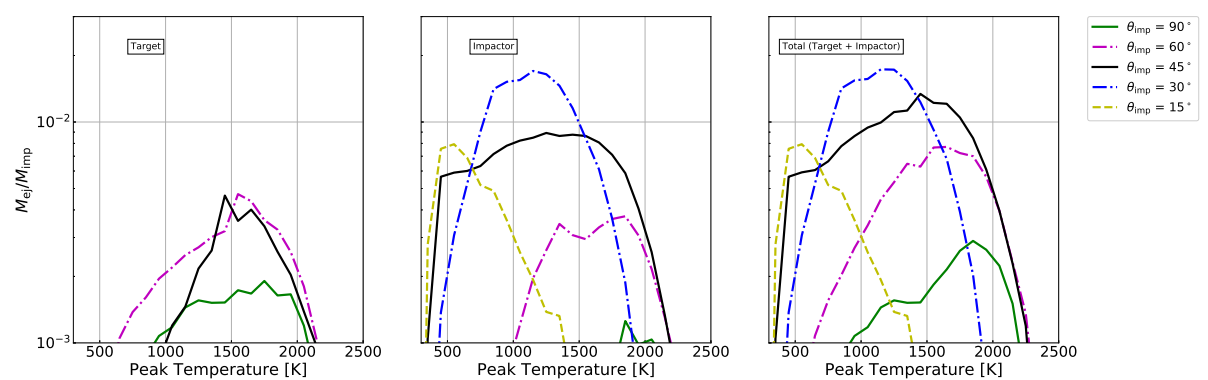

Figure 12: Histogram of peak temperature of ejecta shown in Figures 2(c) and $5\left(v_{\mathrm{ej}} / v_{\mathrm{imp}}>\right.$ 0.5 at $t_{\text {jet }}$ for $\left.v_{\mathrm{imp}}=3 \mathrm{~km} / \mathrm{s}\right)$. Bin size of peak temperature is $100 \mathrm{~K}$. Mass of ejecta $\left(M_{\mathrm{ej}}\right)$ is normalized by mass of impactor $M_{\mathrm{imp}}$.

vaporization) will also increase as the impact becomes more oblique (Melosh and Sonett, 1986, Vickery, 1993). Figure 12 represents the histogram of peak temperature of ejecta shown in Figures $2(\mathrm{c})$ and $5\left(v_{\mathrm{ej}} / v_{\mathrm{imp}}>0.5\right.$ at $t_{\text {jet }}$ for $v_{\mathrm{imp}}$ $=3 \mathrm{~km} / \mathrm{s}$ ). The spatial distribution of fast ejecta colored according to peak temperature (Figure 13) demonstrates that the fastest ejecta, preferentially directed downrange, also reaches the highest temperatures. The peak of each histogram (Figure 12 shifts to higher temperature as the impact angle increases. This seems broadly consistent with laboratory experiments, which showed that the temperature of jetted material increases with impact angles (i.e., vertical impacts produce the hottest jet) (Sugita et al., 1998, Sugita and Schultz, 1999). To track the evolutional history of their temperature (i.e., they have experienced melting), we expand the analyze and quantify the potential amount of jetted melt from our simulations. We define jetted melt as jetted ejecta that exceeds the solidus temperature of dunite (1373K) after the pressure has dropped below 1 bar $\left(10^{5} \mathrm{~Pa}\right)$. When jetted ejecta have reached high pressure, we track them until their pressure becomes lower than this threshold, then examine their temperature as a post-shock temperature to compare with the solidus temperature. Figure 14 shows the total amount of jetted melt $M_{\text {jet }}^{\text {melt }}$ normalized by $M_{\mathrm{imp}}$ as a function of the impact angle. Although the melted ejecta of most cases for $v_{\mathrm{imp}}=3 \mathrm{~km} / \mathrm{s}$ fail to reach $1 \%$ of $M_{\mathrm{imp}}$, likely due to the lower resolution of 

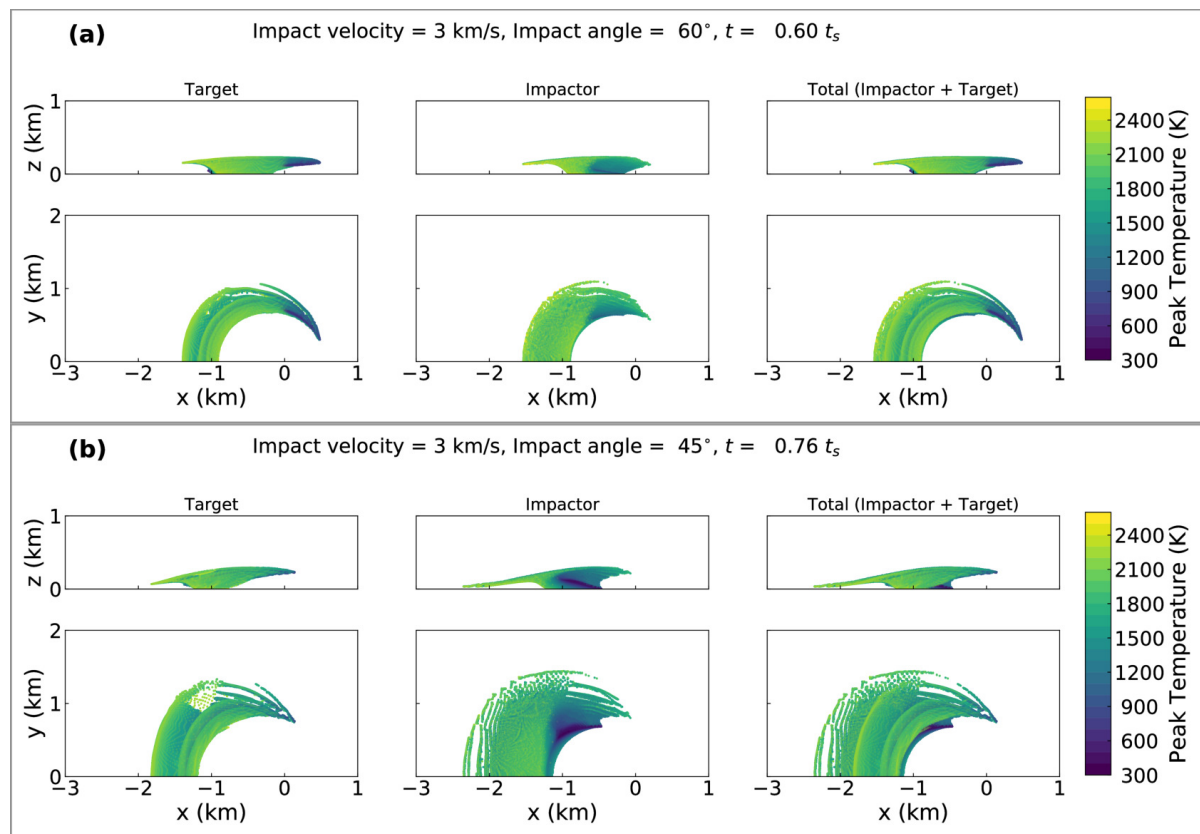

(c) Impact velocity $=3 \mathrm{~km} / \mathrm{s}$, Impact angle $=30^{\circ}, t=0.78 t_{5}$
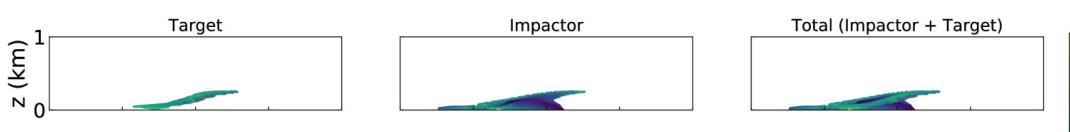

$2400 \underline{\underline{z}}$

2100 뜬
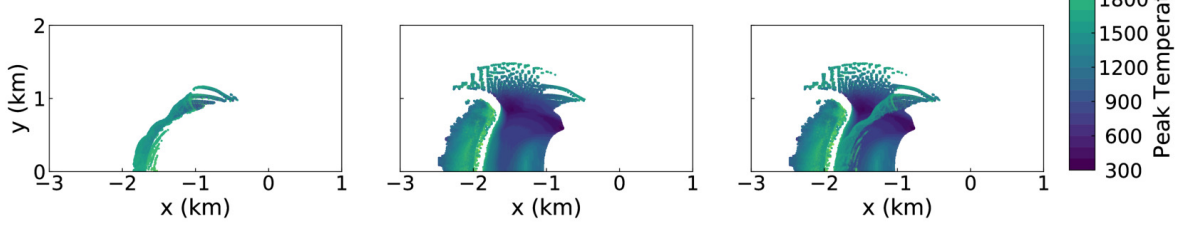

Figure 13: Behavior of ejected material for $\theta_{\mathrm{imp}}=60^{\circ}, 45^{\circ}$, and $30^{\circ}$ (top to bottom). Same viewing geometries and time as Figures 2 (c) and 5 but color represents peak temperature in Kelvin. 

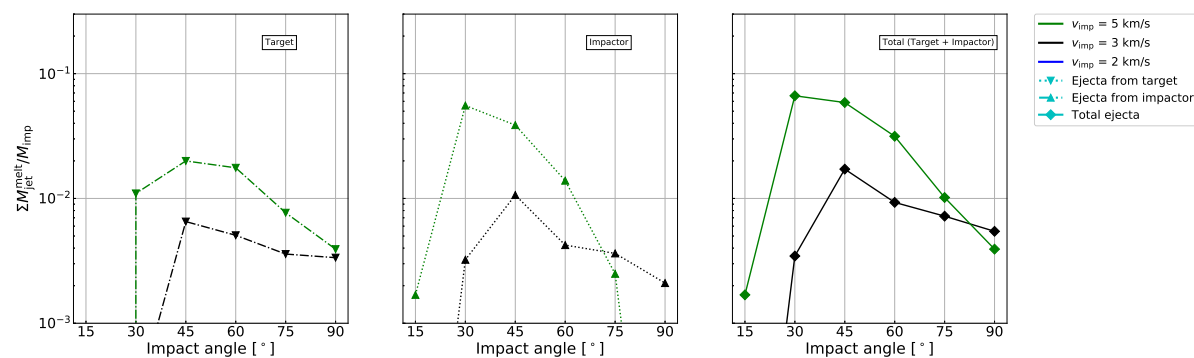

Figure 14: Same as Figure 10 but for $\Sigma M_{\text {jet }}^{\text {melt }}$. Note that $\Sigma M_{\text {jet }}^{\text {melt }}$ of $2 \mathrm{~km} / \mathrm{s}$ is less than $10^{-3}$ and cannot be seen here.

the model run, melted material for $v_{\mathrm{imp}}=5 \mathrm{~km} / \mathrm{s}$ exceeds $1 \%$ of $M_{\mathrm{imp}}$ (green lines in Figure 14, which we consider to be a significant melt mass. We find that melt fraction in jetted ejecta depends on the impact angle, and that melt fraction decreases as impact angles get shallower (Figure 15), which directly conflicts with previous work based on thin plate theory (Vickery, 1993) suggesting that jets produced by grazing impacts have a higher melt content than the jet produced by vertical impacts. However, because the jetted mass produced by moderately oblique impacts is larger than that of vertical impacts (Figure 10), so is the amount of melt. Comparison to high resolution axisymmetric simulations shows that jetted melt in our $v_{\mathrm{imp}}=5 \mathrm{~km} / \mathrm{s}$ is underestimated by a factor of 1.2 similar to the resolution of jetted mass. The same comparison for $v_{\mathrm{imp}}=3 \mathrm{~km} / \mathrm{s}$, however, shows the jetted melt is underestimated by a factor of 2.1 Appendix A). This result is not surprising as previous simulations of Johnson et al. (2015) show that the early fastest-moving portion of the jet, which is more difficult to resolve, is dominated by melt.

If jetted ejecta are melt-dominated (Figures 14 and 15), this may increase the amount of chondrules produced during planetary accretion (e.g., Kieffer, 1975, Johnson et al., 2015; Wakita et al., 2017). Chondrules are millimetersized spherical materials found in primitive meteorites (e.g., Scott and Krot, 2014). While the processes that produce chondrules are still debated, impact jetting during accretionary impacts is one proposed formation mechanism. In this scenario, melt jetted during a relatively low velocity collision may break up 

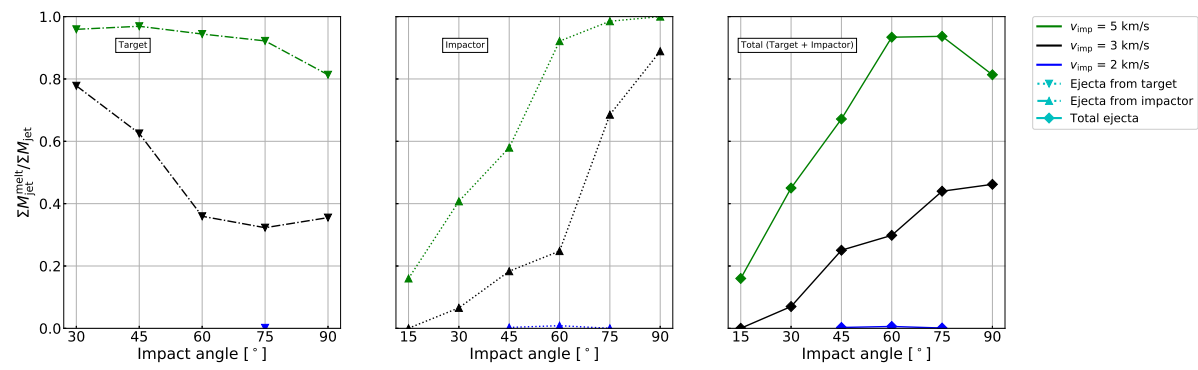

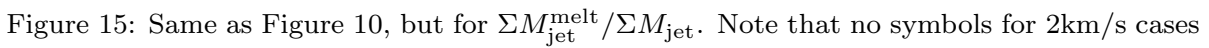
means no jetted melt.

and cool, ultimately becoming chondrules (Johnson et al., 2015, Johnson et al., 2018). The amount of jetted melt from our three-dimensional vertical impact simulations is smaller than previous two-dimensional calculations, likely due to our lower resolution (see also Appendix A). Our work, however, indicates that oblique impacts produce more jetted melt than vertical impacts (Figure 14). Given that oblique impacts occur more frequently (Shoemaker, 1962), they may have enhanced efficiency in chondrule formation in the early Solar System.

Our results also show that the amount of jetted ejecta originating from the impactor increases as impact angles become shallower (Figure 10); this trend suggests that the amount of melted material sufficient to form chondrules will have a greater component of impactor material than head-on impacts at the same impact velocity. Chondrule precursor material is thought to be pristine undifferentiated material (Taylor et al., 1983). While smaller impactors tend to remain undifferentiated, the larger target might be differentiated Gail et al. 2014 Lichtenberg et al., 2018, Wakita et al. 2018), though such larger bodies might have an undifferentiated surface layer (e.g., Weiss and Elkins-Tanton, 2013). Our findings that jetted material produced during oblique impacts is dominated by impactor material may suggest that an undifferentiated impactor can produce chondrules with primitive compositions even if the target is completely differentiated.

Our results may also have implications for the origin of unique distal ejecta 
patterns observed around craters produced by oblique impacts. Distal distribution of ejecta, known as butterfly patterns, are found around elongated craters on Moon and Mars Gault and Wedekind, 1978, Schultz and Lutz-Garihan, 1982). As Figures 5f and 6e indicate, there is a discontinuous distribution of jetted material for $\theta_{\mathrm{imp}}=30^{\circ}$ (the gap around $\sim 30^{\circ}$ of (azimuthal) ejection angle in Fig. 6f, which corresponds to around $(\mathrm{x}, \mathrm{y})=(-2 \mathrm{~km}, 1 \mathrm{~km})$ in Fig. 5.). Slower ejecta released at later stages would be the primary source of an asymmetric ejecta pattern, rather than the earlier jetted ejecta. It is unclear from our work if the asymmetric pattern for fastest ejecta would affect the distribution of later slower ejecta. Additionally, we note the impact velocities explored here are significantly lower than typical impact velocities on the Moon and other terrestrial planets so these comparisons may be limited. s Our results may also explain differences in reflectance in distal ejecta on the Moon as a difference in material composition between target and impactor (Speyerer et al. 2016). While the impact velocity on the Moon ranges over $6 \mathrm{~km} / \mathrm{s}$ to $40 \mathrm{~km} / \mathrm{s}$ (Chyba, 1991; Yue et al. 2013), the escape velocity of the Moon is $2.4 \mathrm{~km} / \mathrm{s}$. Thus, jetted ejecta as defined here will exceed the escape velocity of the Moon. As previously noted, the fastest portions of the jet eject at very low angles and graze the surface. Considering a realistic target with topography and slopes some of this material will impact the surface and be deposited even though it is ejected above escape velocity. Our results for grazing impacts $\left(\theta_{\mathrm{imp}}=15^{\circ}\right)$ record a deficit in ejecta velocity, (around $0.5 v_{\text {imp }}$ ) resulting from the difference in ejecta velocity between ejecta originating from target and impactor (Figures $5 \mathrm{~d}$ and $6 \mathrm{~d})$. If the composition of the impactor differs from that of the target, ejecta of oblique impacts originating from the impactor might produce distal ejecta with different reflectance characteristics than proximal ejecta, which may originate from the target (Speyerer et al., 2016).

Lastly, we assess the influence of resolution on our results by comparing our three dimensional simulations with two-dimensional calculations. Our threedimensional results are in good agreement with two-dimensional simulations at the same resolution of $5 \mathrm{~m}$ or 100 cells per projectile radius (CPPR); however, 
higher resolution is required to produce nearly same amount of jetted material as fully resolved two-dimensional calculations (see Appendix A : The jetted mass in our simulations with 100 CPPR are 60\%-70\% less than that produced in our 1000 CPPR resolution simulations. This finding is consistent with previous work reporting that resolution $>400 \mathrm{CPPR}$ is necessary to resolve high-velocity jetted material based on two-dimensional simulations (Johnson et al. 2014, Wakita et al., 2017, Kurosawa et al., 2018). However, because the cumulative jetted mass produced by oblique impacts is more than twice the amount produced in head-on impacts, our simulations should adequately resolve the influence of impact angle on the mass and the distribution of impactor and target materials in jetted ejecta. It is difficult and computationally expensive to run the threedimensional simulations in high resolution, however, limiting the high-resolution zone as done in this paper will help to tackle this. Our results provide an important step toward understanding jetting during oblique impacts.

\section{Conclusions}

Our simulations of oblique impacts illustrate that the amount of jetted materials and their origin strongly depends on impact angle. For an impact velocity of $3 \mathrm{~km} / \mathrm{s}$, a $45^{\circ}$ impact produces six times the mass of jetted materials relative to a vertical impact. The source of jetted material varies; for $<45^{\circ}$ impacts, most jetted material originates from the impactor; however, target material begins to be the primary source of jetted ejecta at shallower impact angles $\left(75^{\circ}\right.$ and $90^{\circ}$ ). While jetted ejecta from oblique impacts is distributed downrange of the impactor (Figures 2 and 5), grazing impacts (impact angles $<30^{\circ}$ ) have a more discontinuous distribution. Such distribution by grazing impacts may help explain the distal ejecta patterns observed around elongated craters. Additional higher resolution simulations of oblique impacts can provide more accurate estimates of both the amount of jetted ejecta and its melt fraction, a potential source for chondrules. 


\section{Acknowledgments}

We gratefully acknowledge the developers of iSALE-3D, including Gareth Collins, Kai Wünnemann, Dirk Elbeshausen, Boris Ivanov and Jay Melosh. Numerical computations were carried out on the PC cluster and the analysis servers at the Center for Computational Astrophysics, National Astronomical Observatory of Japan. This work was supported by grant 80NSSC20K0422 from the NASA Emerging Worlds program. T. M. D. was funded by STFC Grant ST/S00615/1. We thank anonymous referees whose comments helped to improve this manuscript.

\section{Appendix A. Head-on impacts in two-dimensional calculation}

Here, we compare the results for the mass of jetted material produced in head-on impacts for iSALE-3D to those using the iSALE-2D shock physics code. iSALE-2D is based on the SALE hydrocode Amsden et al., 1980, Wünnemann et al. 2006, Collins et al., 2016, version iSALE-Dellen). The highest resolution we used in iSALE-3D was 5m, with 100 cells per projectile radius (CPPR). For our iSALE-2D runs we include the entire spherical impactor in the high resolution zone, instead of the lower part used in iSALE-3D (see Section 2 and Figure 1). The comparison below demonstrates that the smaller high resolution zone used in our 3D simulations does not affect jetted mass. Figure A.1 shows the evolution of cumulative jetted mass produced from head-on impacts using iSALE-2D with $100 \mathrm{CPPR}$ and $v_{\mathrm{imp}}=3 \mathrm{~km} / \mathrm{s}$. Although the details differ between Figures 8 (iSALE-3D) and A.1 (iSALE-2D), the cumulative jetted mass is $1.1 \%$ of impactor mass for both scenarios. Our results for $\Sigma M_{\text {jet }}$ produced from head-on impacts in iSALE-2D as a function of resolution are are summarized in Figure A.2. Our results for 100 CPPR from iSALE-2D and iSALE-3D with $v_{\mathrm{imp}}=2 \mathrm{~km} / \mathrm{s}$ and $5 \mathrm{~km} / \mathrm{s}$ give similar results $\left(\Sigma M_{\mathrm{jet}} / M_{\mathrm{imp}}\right.$ is $0.68 \%$ and 0.46 $\%$ for iSALE-2D, and $0.62 \%$ and $0.48 \%$ for iSALE-3D, respectively).

Our results show that our simulations using iSALE-2D are well converged at $400 \mathrm{CPPR}$. The jetted mass fraction compared to that produced from 1000 


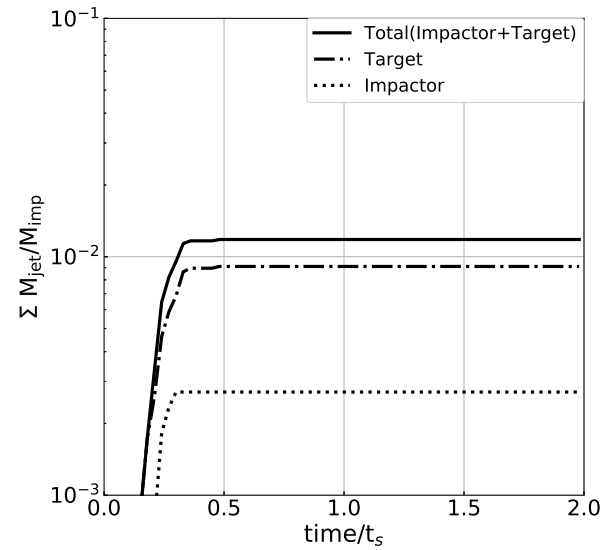

Figure A.1: Same evolution of jetted material over time as in Figure 8 but using iSALE-2D $(\mathrm{CPPR}=100)$.

CPPR are shown in Table A.1. Taking our 1000 CPPR results as fully converged, our 3D head-on impact results with 100 CPPR underestimate the jetted mass by a factor of 1.4 to 1.6, depending on impact velocity. In simulations with $100 \mathrm{CPPR}$, there is little difference between the amount of jetted melt produced in iSALE-2D and iSALE-3D, but they underestimate jetted melt relative to 1000 CPPR simulations by a factor of 2.1 at $3 \mathrm{~km} / \mathrm{s}$ and 1.2 at $5 \mathrm{~km} / \mathrm{s}$ (Figure A.3). Note that jetted melt at $2 \mathrm{~km} / \mathrm{s}$ with 800 and 1000 CPPR exceeds $0.1 \%$ of $M_{\mathrm{imp}}$. It remains unclear whether oblique impact simulations with higher resolution would produce more jetted melt than simulations at 100 CPPR. As Figure 10 illustrates, oblique impacts generate more jetted material than head-on impacts. Therefore, our setting of $100 \mathrm{CPPR}$ may be sufficient to characterize the jetted mass produced by oblique impacts, though not vertical impacts. Regardless, we expect the trends in jetted mass as a function of impact angle and velocity for higher resolution simulations to be similar to those presented here. 

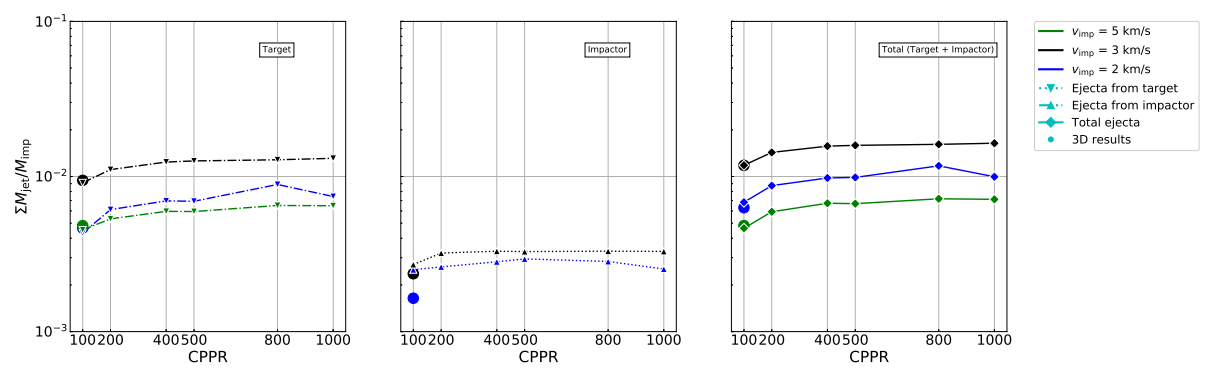

Figure A.2: Cumulative mass of jetted material ( $\left.\Sigma M_{\text {jet }}\right)$ as a function of CPPR for the headon impacts. Color represents different impact velocities: $2 \mathrm{~km} / \mathrm{s}$ (blue), $3 \mathrm{~km} / \mathrm{s}$ (black), and $5 \mathrm{~km} / \mathrm{s}$ (green). The panels depict the total mass $M_{\text {jet }}$ normalized by $M_{\mathrm{imp}}$ from the target (left), the impactor (middle), and the total mass (right), respectively. The head-on impact results from iSALE-3D are plotted as circles. Note that jetted ejecta from the impactor in the case of $5 \mathrm{~km} / \mathrm{s}$ is less than $10^{-3}$ (middle panel).
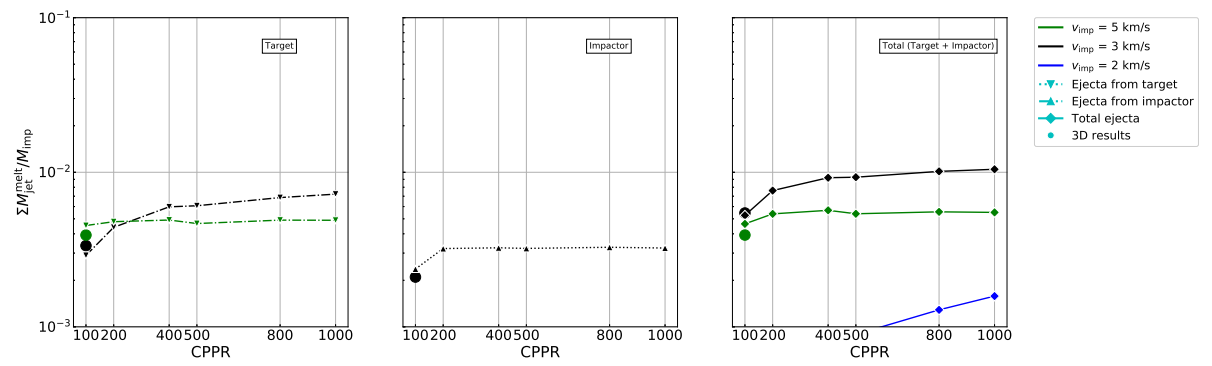

Figure A.3: Same as Figure A.2 but for $\Sigma M_{\text {jet }}^{\text {melt }}$. Note that jetted melt from the target at 2 $\mathrm{km} / \mathrm{s}$ and the impactor at $2 \mathrm{~km} / \mathrm{s}$ and $5 \mathrm{~km} / \mathrm{s}$ are less than $10^{-3}$. 
Table A.1: The jetted mass fraction ratio to that from $\mathrm{CPPR}=1000$ *

\begin{tabular}{lccccccl}
\hline \hline$v_{\text {imp }}$ & CPPR $=100(3 \mathrm{D})$ & $100(2 \mathrm{D})$ & 200 & 400 & 500 & 800 & 1000 \\
\hline $2 \mathrm{~km} / \mathrm{s}$ & 0.63 & 0.68 & 0.87 & 0.98 & 0.99 & 1.1 & $1.0\left(9.9 \times 10^{-3}\right)$ \\
$3 \mathrm{~km} / \mathrm{s}$ & 0.72 & 0.72 & 0.87 & 0.95 & 0.96 & 0.98 & $1.0\left(1.6 \times 10^{-2}\right)$ \\
$5 \mathrm{~km} / \mathrm{s}$ & 0.67 & 0.65 & 0.83 & 0.94 & 0.93 & 1.0 & $1.0\left(7.1 \times 10^{-3}\right)$ \\
\hline
\end{tabular}

* These values are respected to the jetted mass fraction of $\mathrm{CPPR}=1000$ (see Figure A.2. Absolute values of $\mathrm{CPPR}=1000$ are shown in bracket.

\section{References}

Amsden, A., Ruppel, H., Hirt, C., 1980. Sale: a simplified ale computer program for fluid flow at all speeds. Los Alamos National Laboratories Report doi:10. $2172 / 5176006$.

Benz, W., Cameron, A., Melosh, H., 1989. The origin of the moon and the single-impact hypothesis iii. Icarus 81, 113 - 131 . URL: http: //www.sciencedirect.com/science/article/pii/0019103589901292, doi:https://doi.org/10.1016/0019-1035(89)90129-2.

Birkhoff, G., MacDougall, D.P., Pugh, E.M., Taylor, Geoffrey, S., 1948. Explosives with Lined Cavities. Journal of Applied Physics 19, 563-582. doi:10.1063/1.1698173.

Bottke, W.F., Love, S.G., Tytell, D., Glotch, T., 2000. Interpreting the elliptical crater populations on mars, venus, and the moon. Icarus 145, 108 - 121. URL: http://www.sciencedirect.com/science/article/pii/ S0019103599963236, doi https://doi.org/10.1006/icar.1999.6323.

Burchell, M.J., Mackay, N.G., 1998. Crater ellipticity in hypervelocity impacts on metals. Journal of Geophysical Research: Planets 103, 22761-22774. URL: https://agupubs.onlinelibrary. wiley.com/doi/abs/10.1029/98JE02143, doi 10.1029/98JE02143, arXiv:https://agupubs.onlinelibrary.wiley.com/doi/pdf/10.1029/98JE02143. 
Chyba, C.F., 1991. Terrestrial mantle siderophiles and the lunar impact record. Icarus 92, 217 - 233. URL: http://www.sciencedirect. com/science/article/pii/001910359190047W, doi/https://doi.org/10. 1016/0019-1035(91)90047-W.

Collins, G., Melosh, H., Wünnemann, K., 2011. Improvements to the $\epsilon-\alpha$ porous compaction model for simulating impacts into high-porosity solar system objects. International Journal of Impact Engineering 38, 434-439. URL: http: //wWw.sciencedirect.com/science/article/pii/S0734743X10001594, doi:https://doi.org/10.1016/j.ijimpeng.2010.10.013. hypervelocity Impact selected papers from the 2010 Symposium.

Collins, G.S., Elbeshausen, D., Davison, T.M., Wünnemann, K., Ivanov, B., Melosh, H.J., 2016. iSALE-Dellen manual URL: https: //figshare.com/articles/iSALE-Dellen_manual/3473690, doi 10.6084/ m9.figshare.3473690.v2

Collins, G.S., Melosh, H.J., Ivanov, B.A., 2004. Modeling damage and deformation in impact simulations. Meteoritics and Planetary Science 39, 217-231. doi $10.1111 / j .1945-5100.2004 . t b 00337 . x$.

Davison, T.M., 2010. Numerical modelling of heat generation in porous planetesimal collisions. PhD thesis, Imperial College London, London, U. K. .

Davison, T.M., Ciesla, F.J., Collins, G.S., Elbeshausen, D., 2014. The effect of impact obliquity on shock heating in planetesimal collisions. Meteoritics and Planetary Science 49, 2252-2265. doi 10.1111/maps.12394.

Davison, T.M., Collins, G.S., Bland, P.A., 2016. Mesoscale Modeling of Impact Compaction of Primitive Solar System Solids. ApJ 821, 68. doi 10.3847/ 0004-637X/821/1/68, arXiv:1603.00376

Davison, T.M., Collins, G.S., Elbeshausen, D., Wünnemann, K., Kearsley, A., 2011. Numerical modeling of oblique hypervelocity impacts on strong ductile 
targets. Meteoritics and Planetary Science 46, 1510-1524. doi:10.1111/j. $1945-5100.2011 .01246 \cdot \mathrm{x}$

Elbeshausen, D., Wünnemann, K., Collins, G.S., 2009. Scaling of oblique impacts in frictional targets: Implications for crater size and formation mechanisms. Icarus 204, 716-731. doi $10.1016 / j$.icarus.2009.07.018

Gail, H.P., Trieloff, M., Breuer, D., Spohn, T., 2014. Early Thermal Evolution of Planetesimals and Its Impact on Processing and Dating of Meteoritic Material. p. 571. doi 10.2458/azu_uapress_9780816531240-ch025, arXiv:1312.3509

Gault, D.E., Wedekind, J.A., 1978. Experimental studies of oblique impact. Lunar and Planetary Science Conference Proceedings 3, 3843-3875.

Hasegawa, Y., Turner, N.J., Masiero, J., Wakita, S., Matsumoto, Y., Oshino, S., 2016. Forming Chondrites in a Solar Nebula with Magnetically Induced Turbulence. ApJ 820, L12. doi 10.3847/2041-8205/820/1/L12, arXiv:1603.00086.

Hirt, C., Amsden, A., Cook, J., 1974. An arbitrary lagrangianeulerian computing method for all flow speeds. Journal of Computational Physics 14, 227 - 253. URL: http://www.sciencedirect. com/science/article/pii/0021999174900515, doi/https://doi.org/10. 1016/0021-9991(74)90051-5.

Ivanov, B., Deniem, D., Neukum, G., 1997. Implementation of dynamic strength models into 2d hydrocodes: Applications for atmospheric breakup and impact cratering. International Journal of Impact Engineering 20, 411 - 430. URL: http://www.sciencedirect.com/science/article/ pii/S0734743X97875112, doi:https://doi.org/10.1016/S0734-743X(97) 87511-2. hypervelocity Impact Proceedings of the 1996 Symposium.

Johnson, B.C., Bowling, T.J., Melosh, H.J., 2014. Jetting during vertical im- 
pacts of spherical projectiles. Icarus $238,13-22$. doi $10.1016 / j$.icarus. 2014.05 .003 .

Johnson, B.C., Ciesla, F.J., Dullemond, C.P., Melosh, H.J., 2018. Formation of Chondrules by Planetesimal Collisions. Cambridge University Press. Cambridge Planetary Science, pp. 343-360. doi 10.1017/9781108284073.013.

Johnson, B.C., Melosh, H.J., 2014. Formation of melt droplets, melt fragments, and accretionary impact lapilli during a hypervelocity impact. Icarus 228, 347-363. doi:10.1016/j.icarus.2013.10.022

Johnson, B.C., Minton, D.A., Melosh, H.J., Zuber, M.T., 2015. Impact jetting as the origin of chondrules. Nature 517, 339-341. doi 10.1038/nature14105

Kieffer, S.W., 1975. Droplet chondrules. Science 189, 333-340. URL: https://science.sciencemag.org/ content/189/4200/333, doi $10.1126 /$ science.189.4200.333 arXiv:https://science.sciencemag.org/content/189/4200/333.full.pdf.

Kieffer, S.W., 1977. Impact conditions required for formation of melt by jetting in silicates., in: Roddy, D.J., Pepin, R.O., Merrill, R.B. (Eds.), Impact and Explosion Cratering: Planetary and Terrestrial Implications, pp. 751-769.

Kokubo, E., Ida, S., 2000. Formation of Protoplanets from Planetesimals in the Solar Nebula. Icarus 143, 15-27. doi:10.1006/icar.1999.6237.

Kurosawa, K., Genda, H., 2018. Effects of Friction and Plastic Deformation in Shock-Comminuted Damaged Rocks on Impact Heating. Geophys. Res. Lett. 45, 620-626. doi 10.1002/2017GL076285, arXiv:1801.01100

Kurosawa, K., Nagaoka, Y., Senshu, H., Wada, K., Hasegawa, S., Sugita, S., Matsui, T., 2015. Dynamics of hypervelocity jetting during oblique impacts of spherical projectiles investigated via ultrafast imaging. Journal of Geophysical Research (Planets) 120, 1237-1251. doi:10.1002/2014JE004730. 
Kurosawa, K., Okamoto, T., Genda, H., 2018. Hydrocode modeling of the spallation process during hypervelocity impacts: Implications for the ejection of Martian meteorites. Icarus 301, 219-234. doi 10.1016/j.icarus.2017. 09.015, arXiv:1709.00561.

Lichtenberg, T., Golabek, G.J., Dullemond, C.P., Schönbächler, M., Gerya, T.V., Meyer, M.R., 2018. Impact splash chondrule formation during planetesimal recycling. Icarus 302, 27-43. doi:10.1016/j.icarus.2017.11.004, arXiv:1711.02103

McKinnon, W.B., 1989a. Impact jetting of water ice, with application to the accretion of icy planetesimals and Pluto. Geophys. Res. Lett. 16, 1237-1240. doi:10.1029/GL016i011p01237.

McKinnon, W.B., 1989b. On the Origin of the Pluto-Charon Binary. ApJ 344, L41. doi:10.1086/185526.

Melosh, H.J., Ryan, E.V., Asphaug, E., 1992. Dynamic fragmentation in impacts - Hydrocode simulation of laboratory impacts. J. Geophys. Res. 97, 14. doi $10.1029 / 92 \mathrm{JE} 01632$

Melosh, H.J., Sonett, C.P., 1986. When worlds collide: jetted vapor plumes and the Moon's origin., in: Hartmann, W.K., Phillips, R.J., Taylor, G.J. (Eds.), Origin of the Moon, pp. 621-642.

Pierazzo, E., Melosh, H.J., 2000. Hydrocode modeling of oblique impacts: The fate of the projectile. Meteoritics and Planetary Science 35, 117-130. doi:10. 1111/j.1945-5100.2000.tb01979.x.

Schultz, P.H., D'Hondt, S., 1996. Cretaceous-Tertiary (Chicxulub) impact angle and its consequences. Geology 24, 963-967. URL: https://doi.org/10.1130/0091-7613(1996)024<0963:CTCIAA>2 . 3.CO;2 doi 10.1130/0091-7613(1996)024<0963:CTCIAA>2.3.CO;2, arXiv:https://pubs.geoscienceworld.org/geology/article-pdf/24/11/963/3516302/i0091-7613-2 
Schultz, P.H., Gault, D.E., 1990. Decapitated Impactors in the Laboratory and on the Planets, in: 21st Lunar and Planetary Science Conference, p. 49.

Schultz, P.H., Gault, D.E., 1990. Prolonged global catastrophes from oblique impacts, in: Global Catastrophes in Earth History; An Interdisciplinary Conference on Impacts, Volcanism, and Mass Mortality. Geological Society of America. URL: https://doi.org/10.1130/SPE247-p239, doi10.1130/ SPE247-p239.

Schultz, P.H., Lutz-Garihan, A.B., 1982. Grazing Impacts on Mars: a Record of Lost Satellites. Lunar and Planetary Science Conference Proceedings 87, 84-A96. doi:10.1029/JB087iS01p00A84.

Scott, E.R.D., Krot, A.N., 2014. Chondrites and Their Components. pp. 65-137.

Shoemaker, E.M., 1962. Interpretation of lunar craters. Academic Press. pp. $283-359$.

Speyerer, E.J., Povilaitis, R.Z., Robinson, M.S., Thomas, P.C., Wagner, R.V., 2016. Quantifying crater production and regolith overturn on the Moon with temporal imaging. Nature 538, 215-218. doi $10.1038 /$ nature19829

Stöffler, D., Artemieva, N.A., Wünnemann, K., Reimold, W.U., Jacob, J., Hansen, B.K., Summerson, I.A.T., 2013. Ries crater and suevite revisited-Observations and modeling Part I: Observations. Meteoritics and Planetary Science 48, 515-589. doi:10.1111/maps.12086.

Sugita, S., Schultz, P.H., 1999. Spectroscopic characterization of hypervelocity jetting: Comparison with a standard theory. J. Geophys. Res. 104, 3082530846. doi $10.1029 / 1999$ JE001061.

Sugita, S., Schultz, P.H., Adams, M.A., 1998. Spectroscopic measurements of vapor clouds due to oblique impacts. Journal of Geophysical Research: Planets 103, 19427-19441. URL: https://agupubs.onlinelibrary.wiley.com/ doi/abs/10.1029/98JE02026, doi:https://doi.org/10.1029/98JE02026, arXiv:https://agupubs.onlinelibrary.wiley.com/doi/pdf/10.1029/98JE02026 
Taylor, G.J., Scott, E.R.D., Keil, K., 1983. Cosmic setting for chondrule formation, in: Chondrules and their Origins, pp. 262-278.

Vickery, A.M., 1993. The Theory of Jetting: Application to the Origin of Tektites. Icarus 105, 441-453. doi 10.1006/icar.1993.1140.

Wakita, S., Hasegawa, Y., Nozawa, T., 2018. Abundances of Ordinary Chondrites in Thermally Evolving Planetesimals. ApJ 863, 100. doi 10.3847/ 1538-4357/aad0a2, arXiv:1806.11252.

Wakita, S., Matsumoto, Y., Oshino, S., Hasegawa, Y., 2017. Planetesimal Collisions as a Chondrule Forming Event. ApJ 834, 125. doi 10.3847/1538-4357/ 834/2/125, arXiv:1611.05511.

Walsh, J.M., Shreffler, R.G., Willig, F.J., 1953. Limiting Conditions for Jet Formation in High Velocity Collisions. Journal of Applied Physics 24, 349359. doi $10.1063 / 1.1721278$.

Weiss, B.P., Elkins-Tanton, L.T., 2013. Differentiated Planetesimals and the Parent Bodies of Chondrites. Annual Review of Earth and Planetary Sciences 41, 529-560. doi 10.1146/annurev-earth-040610-133520

Wünnemann, K., Collins, G.S., Melosh, H.J., 2006. A strain-based porosity model for use in hydrocode simulations of impacts and implications for transient crater growth in porous targets. Icarus $180,514-527$. doi $10.1016 / j$. icarus.2005.10.013.

Yang, W., Ahrens, T.J., 1995. Impact jetting of geological materials. Icarus 116, 269 - 274. URL: http://www.sciencedirect.com/science/article/ pii/S0019103585711256, doi/https://doi.org/10.1006/icar.1995.1125

Yue, Z., Johnson, B.C., Minton, D.A., Melosh, H.J., Di, K., Hu, W., Liu, Y., 2013. Projectile remnants in central peaks of lunar impact craters. Nature Geoscience 6, 435-437. URL: https://doi.org/10.1038/ngeo1828, doi:10. $1038 /$ ngeo1828. 